\title{
Involvement of hemeoxygenase-1 in di(2-ethylhexyl) phthalate (DEHP)-induced apoptosis of Neuro-2a cells
}

\author{
Kyaw Htet Aung ${ }^{1}$, Tin-Tin Win-Shwe ${ }^{2}$, Moeko Kanaya ${ }^{1}$, Hirohisa Takano ${ }^{3}$ \\ and Shinji Tsukahara ${ }^{1}$

\begin{abstract}
${ }^{1}$ Division of Life Science, Graduate School of Science and Engineering, Saitama University, 255 Shimo-Okubo, Sakura-ku, Saitama City, Saitama 338-8570, Japan

${ }^{2}$ Center for Environmental Health Sciences, National Institute for Environmental Studies, 16-2 Onogawa, Tsukuba, Ibaraki 305-8506, Japan
\end{abstract} \\ ${ }^{3}$ Department of Environmental Engineering, Graduate School of Engineering, Kyoto University, \\ C Cluster, Kyoto-Daigaku-Katsura, Nishikyo-ku, Kyoto 615-8540, Japan
}

(Received December 4, 2013; Accepted January 25, 2014)

\begin{abstract}
A widely-used plasticizer di(2-ethylhexyl) phthalate (DEHP) is known to induce apoptosis in neurons, although the mechanisms responsible for DEHP-induced apoptosis is not well explored yet. We recently showed that exposure to DEHP increases the expression of hemeoxygenase (HO)-1, an oxidative stress related enzyme, in the mice brain. In this study, we investigated whether HO-1 is involved in DEHP-induced apoptosis using a mouse neuroblastoma cell line Neuro-2a, which forcibly express SCAT3, a fluorescent indicator of caspase-3 activity. The doses of DEHP at 1,10 or $100 \mu \mathrm{M}$ were used in the present study to mimic the level of human exposure to DEHP. Live image analysis of SCAT3-expressing Neuro-2a cells revealed that caspase-3 activity in the cells was significantly increased by DEHP at $100 \mu \mathrm{M}$ but not 1 or $10 \mu \mathrm{M}$. We measured HO-1 mRNA level in Neuro-2a cells exposed to DEHP and found significant increase in HO-1 mRNA level by DEHP at $100 \mu \mathrm{M}$ but not 1 or $10 \mu \mathrm{M}$. Live image analysis of SCAT3-expresisng Neuro-2a cells was further performed to determine the effects of HO-1 siRNA in DEHP-induced apoptosis via caspase-3 activation. We found that knockdown of HO-1 gene nullifies the effects of DEHP to activate caspase-3. These results suggest that HO-1 is involved in DEHP-induced apoptosis. Moreover, this study demonstrates that high-dose DEHP exposure induces caspase-3-dependent apoptosis, which is at least partially mediated by the up-regulation of HO-1 gene, in Neuro-2a cells.
\end{abstract}

Key words: DEHP, Apoptosis, Hemeoxygenase-1, Caspase-3, Neuro-2a, Live imaging

\section{INTRODUCTION}

Phthalates are widely used as plasticizers in the production of goods made of polyvinylchloride (PVC) such as food packaging, toys, cosmetics, medical devices, etc. Exposure to phthalates is thought to be increasing as the result of ingestion, inhalation, and dermal contact via plastic-made products. It is therefore raising concerns about the health risks of phthalates. Animal studies have shown that developmental exposure to di(2-ethylhexyl) phthalate (DEHP), a commonly used phthalate plasticizers, causes reproductive tract abnormalities compatible with the disruption of androgen-dependent development and impaired testicular functions in rodents (Klinefelter et al., 2012;
Xi et al., 2011). DEHP is also known to have developmental neurotoxicity, because spontaneous motor activity in young adult rats is increased by neonatal exposure to DEHP (Masuo et al., 2004). Moreover, it was reported that perinatal DEHP exposure impaired motor coordination of adolescent rats (Arcadi et al., 1998) and reduced the number of dopaminergic neurons in the midbrain and brain weight of adolescent mice (Tanida et al., 2009). Epidemiological studies have demonstrated that developmental exposure to phthalate decreases the intelligence of school-age children (Cho et al., 2010) and increases the risk of neuropsychiatric disorders (Testa et al., 2012). Accordingly, it is suspected that phthalate exposure causes detrimental effects on the development of the brain.

Correspondence: Tin Tin Win Shwe (E-mail: tin.tin.win.shwe@nies.go.jp) 
However, little is known about the mechanism underlying DEHP-induced developmental neurotoxicity.

Apoptosis, which can often be seen when cells are exposed to toxic chemicals, is partly considered to be the mechanisms underlying the pathogenesis of diseases including neurodevelopmental and neurodegenerative disorders. It was reported that apoptosis occurs in a mouse neuroblastoma cell line Neuro-2a with exposure to DEHP (Lin et al., 2011) and in neurons differentiated from mouse embryonic stem cells with exposure to mono (2-ethylhexyl) phthalate (MEHP), a metabolite of DEHP (Lim et al., 2009). Moreover these reports showed that DEHP and MEHP stimulate the activity of caspase-3 (Lin et al., 2011; Lim et al., 2009). Caspase-3 is a major executioner protease of apoptotic processes in the central nervous system (Pettmann and Henderson, 1998). An abnormal increase in the number of cells with consequent enlargement of brain, morphogenetic change and premature death occurred in the mice lacking caspase-3 gene (Kuida et al., 1996). These indicate that caspase-3 plays essential roles in brain development and that apoptosis of neurons through activation of caspase- 3 normally occurs in developing brain to construct appropriate brain structures with removing unadapted neurons. Taken together, it was suggested that exposure to DEHP and its metabolites during developmental period exert deleterious effects on brain by inducing apoptosis through caspase- 3 activation at an abnormal level.

Heme oxygenase 1 (HO-1) is an enzyme that catalyzes the degradation of heme to biliverdin, iron, and carbon monoxide (CO). The expression of HO-1 is induced in response to stress such as oxidative stress, hypoxia, heavy metals, and cytokines (Imuta et al., 2007; Kietzmann et al., 2003; Alam et al., 2000; Elbirt et al., 1998; Hambleton et al., 1996; Mitani et al., 1993). Several lines of evidence have revealed that HO-1 has dual effects under oxidative stress (Suttner and Dennery, 1999; Suttner et al., 1999; da Silva et al., 1996). It was reported that induction of HO-1 protects cells against oxidative injury through antioxidant activities of bilirubin (Ryter et al., 2007; Otterbein et al., 1995). In contrast, HO-1 is also known to mediate adverse effects of oxidative stress, because there are reports showing that overexpression of HO-1 increase the cellular concentration of reactive iron, followed by induction of apoptosis in neurons and fibroblasts (Suttner and Dennery, 1999; Hsieh et al., 2008a). Thus, HO-1 has cytoprotective and anti-inflammatory actions to protect cells against oxidative stress and cytotoxic actions killing cells by apoptosis, although it remains to determine the mechanisms responsible for switching HO-1 actions.
We recently reported that DEHP exposure increases the expression of HO-1 in the brain of mice (Win-Shwe et al., 2013). This suggests that HO-1 is related to DEHP-induced neurotoxicity. However, it is not clear how HO-1 is involved in DEHP-induced neurotoxicity, because HO-1 has not only cytoprotective and anti-inflammatory actions but also cytotoxic actions. In the present study, we aimed to determine the role of HO-1 in DEHP-induced apoptosis using Neuro-2a cells, a mouse neuroblastoma cell line. We previously showed that live imaging of in vitro cultured cells is a useful tool for toxic evaluation of environmental chemicals (Aung et al., 2013; Koike-Kuroda et al., 2010). First, we therefore monitored Neuro-2a cells expressing SCAT3, a fluorescent indicator of caspase-3 activity, by live imaging and examined the effects of DEHP in caspase-3-dependent apoptosis. Next we measured the mRNA levels of HO-1 in Neuro-2a cells exposed to DEHP to examine whether HO-1 gene expression in neuronal cells is changed by DEHP exposure. Finally, we performed live imaging analysis of SCAT3-expressing Neuro-2a cells to investigate the effects of knockdown of HO-1 gene by its siRNA in DHEP-induced apoptosis.

\section{MATERIALS AND METHODS}

\section{Cell culture and reagent preparation}

Neuro-2a cells were cultured in minimum essential medium (MEM; Gibco-Invitrogen, Carlsbad, CA, USA) supplemented with $0.1 \mathrm{mM}$ non-essential amino acid (NEAA; Gibco-Invitrogen) and 10\% fetal bovine serum (FBS; Gibco-Invitrogen), hereafter referred to as the cell maintenance medium. Cells were passaged every 2-3 days, and a passage number of 7-20 was used for all the experiments.

For each experiment, Neuro-2a cells were cultured in a medium containing MEM (Gibco-Invitrogen) supplemented with $0.1 \mathrm{mM}$ NEAA (Gibco-Invitrogen), 1\% FBS (Gibco-Invitrogen), and $0.3 \mathrm{mM} \mathrm{N6}$, 2'-O-dibutyryl-adenosine cyclic monophosphate sodium salt (dibutyryl cyclic AMP; Sigma-Aldrich, St. Louis, MO, USA), hereafter referred to as the experimental medium. Neuro-2a cells cultured with the experimental medium were simultaneously exposed to DEHP at a concentration of 0 , 1,10 , or $100 \mu \mathrm{M}$. The doses of DEHP used in this study are relevant to the environmental level of DEHP exposed to human. According to the study of Latini et al., 2003, the concentration of DEHP in the blood of human umbilical cord is approximately $1 \mu \mathrm{g} / \mathrm{ml}$ that is equivalent to a concentration of $2.5 \mu \mathrm{M}$. Moreover, neonatal exchange transfusion with PVC-made catheters could increase the blood level of DEHP to the range from 13.2 to 
DEHP and neurotoxicity

$84.9 \mu \mathrm{g} / \mathrm{ml}$ (approximately 30 to $200 \mu \mathrm{M}$ ) (Kavlock et al., 2002). We therefore considered the doses of DEHP at 1,10 , and $100 \mu \mathrm{M}$ used in this study as low-, moderateand high-dose of DEHP exposure, respectively. Because of the poor solubility of DEHP in water, DEHP was dissolved in dimethyl sulfoxide (DMSO) at a concentration of $100 \mathrm{mM}$. DEHP dissolved in DMSO was then diluted with the experimental medium to yield the above-mentioned doses. The medium used for the control group only contained DMSO, and the final concentration of DMSO in all experimental groups was set at $0.1 \%$.

\section{Cell viability assay}

The effects of DEHP on the viability of Neuro-2a cells were assessed using a colorimetric assay based on the reduction of tetrazolium salt to formazan by mitochondrial dehydrogenase activity. Neuro-2a cells were seeded on 96-well plates ( 1 x $10^{4}$ cells/well), which were pre-coated with $0.25 \%$ polyethyleneimine (Sigma-Aldrich), and cultured in $100 \mu \mathrm{l}$ of cell maintenance media. The plate was then kept in an incubator overnight at $37^{\circ} \mathrm{C}$ and in an atmosphere of $5 \% \mathrm{CO}_{2}$. On the next day, the cell maintenance medium was changed to fresh experimental medium containing DEHP at a concentration of $0,1,10$, or $100 \mu \mathrm{M}$. Cell viability was assessed at $24 \mathrm{hr}$ after the initiation of DEHP by the addition of $10 \mu \mathrm{l}$ of tetrazolium reagent WST-1 (Roche Applied Bioscience, Indianapolis, IN, USA) to each well of the culture plate followed by incubation for $3 \mathrm{hr}$ at $37^{\circ} \mathrm{C}$ and $5 \% \mathrm{CO}_{2}$. Absorbance at $450 \mathrm{~nm}$ was then measured using a reference wavelength of $650 \mathrm{~nm}$. The absorbance at $650 \mathrm{~nm}$ was subtracted from the absorbance at $450 \mathrm{~nm}$, and this value was then expressed as a percentage of the value obtained for Neuro-2a cells from the control group, the viability of which was set at $100 \%$. Each experimental group was performed in triplicate for each experiment, and the data was obtained from four independent experiments.

\section{DNA transfection and live imaging analysis}

Neuro-2a cells (2-4 x $10^{4}$ cells/well) were seeded onto an 8 -well chambered glass coated with $0.25 \%$ polyethyleneimine (Sigma-Aldrich) and cultured with $300 \mu \mathrm{l}$ of cell maintenance medium. The cells were then transfected with pcDNA SCAT3 vector, provided by Dr. M. Miura (The University of Tokyo, Tokyo, Japan), using FuGENE HD transfection reagents (Promega, Madison, WI, USA). SCAT3 contains a caspase-3 cleavage peptide fused to enhanced cyan fluorescence protein (ECFP) and Venus, an altered green fluorescence protein, and the life and death of cell can be monitored by measuring the fluorescent intensities of ECFP and Venus (Takemoto et al., 2003).
According to the manufacturer's protocol, we transfected Neuro-2a cells with $0.25 \mu \mathrm{g}$ of pcDNA-SCAT3 in $300 \mu \mathrm{l}$ of cell maintenance medium in an atmosphere containing $5 \% \mathrm{CO}_{2}$ at $37^{\circ} \mathrm{C}$ for $8 \mathrm{hr}$. After transfection, Neuro-2a cells were washed with phosphate-buffered saline to remove the transfection reagent and were kept in $300 \mu \mathrm{l}$ of cell maintenance medium in an atmosphere containing $5 \% \mathrm{CO}_{2}$ at $37^{\circ} \mathrm{C}$ until the start of live imaging.

One or two days after transfection, SCAT3-expressing Neuro-2a cells were cultured in the experimental medium with or without $\operatorname{DEHP}(0,1,10$, or $100 \mu \mathrm{M})$. One hour after the initiation of DEHP exposure, live imaging of SCAT3-expressing Neuro-2a cells was started using a fluorescence time-lapse microscope (BioRevo9000; Keyence Co., Osaka, Japan). SCAT3expressing Neuro-2a cells were placed in an incubation chamber assembled within the microscope (Microscope Incubation System INU-KI-F1; Tokai Hit, Shizuoka, Japan), in which the temperature and gas concentration were controlled at $37^{\circ} \mathrm{C}$ and $5 \% \mathrm{CO}_{2}$, respectively. A 440AF21 excitation filter, a 455DRLP dichroic mirror, and two emission filters (480AF30 for ECFP and 535AF25 for Venus; Opto Science Inc., Tokyo, Japan) were used for fluorescent time-lapse imaging. Images of the SCAT3-expressing Neuro-2a cells were captured using an objective lens (Plan Fluor ELWD DM 20xC, NA 0.45; Nikon, Tokyo, Japan) and a CCD camera attached to the fluorescent microscope every $30 \mathrm{~min}$ within a $24-\mathrm{hr}$ period. Four independent live imaging experiments were performed in this study.

To examine the effect of DEHP exposure on apoptosis in SCAT3-expressing Neuro-2a cells, the fluorescence intensity of ECFP and Venus emissions was measured using digital image data, as previously described (Aung et al., 2013; Koike-Kuroda et al., 2010). Briefly, the colors representing ECFP (cyan blue) and Venus (yellow green) in the digital images of the SCAT3-expressing Neuro-2a cells were converted into monochromatic color images of red and green, respectively. These images were merged at each time point to measure the fluorescence intensities of ECFP and Venus simultaneously. After the brightness of the red color for ECFP and the green color for Venus was measured, the ECFP/Venus emission ratio was calculated by dividing the brightness value of the red color by that of the green color. The calculated values for each time point were calibrated with the ratio at the same time in the control group, which was set at a value of 1 .

In the experiment for evaluating the effects of $\mathrm{HO}-1$ gene knockdown in DEHP-induced apoptosis, Neuro-2a cells were transfected with the SCAT3 expression vector using the above-mentioned method and were subse- 
quently transfected with rodent HO-1 siRNA (5 - GCC GAG AAU GCU GAG UUC A-3') (Hsieh et al., 2008b) or control nucleotide sequences (control siRNA: 5 '-UAA GGC UAU GAA GAG AUA C-3`) (Yan et al., 2007). siRNA transfection was performed using Lipofectamine RNAiMAX reagent (Gibco-Invitrogen) according to the manufacturer's protocol. Two or three days after siRNA transfection, Neuro-2a cells were exposed to DEHP (0 or $100 \mu \mathrm{M})$, and live imaging analyses were then performed using the above-mentioned procedures.

\section{Quantification of specific-gene expression}

The effects of DEHP on the mRNA level of HO-1 and a housekeeping gene glyceraldehyde-3-phosphate dehydrogenase (GAPDH) were measured by a real-time PCR analysis. The mRNA level of GAPDH was used to normalize HO-1 gene expression. The specific primer sets for HO-1 (forward primer: 5 '-CAGATGGCGTCACTTCGT-3 '; reverse primer: 5`-ATTCCCA CTGCC ACTGTT-3`) and GAPDH (forward primer: 5 '-CACTGCCACCCAGAAGA-3`; reverse primer: 5 '-TCCACGA CGGACACATT-3`) were designed with LightCycler Probe Design Software 2.0 (Roche Diagnostics, Mannheim, Germany) using the mRNA sequences downloaded from the genetic sequence database at the National Center for Biotechnical Information (NCBI) Genbank to obtain a melting temperature of $\approx 60^{\circ} \mathrm{C}$, a GC content of $\approx 50 \%$, and amplicon lengths from 150 to $200 \mathrm{bp}$.

Neuro-2a cells seeded on $0.25 \%$ polyethyleneiminecoated 12 -well plates $\left(10^{5}\right.$ cells/well $)$ were grown in the cell maintenance medium in an atmosphere of $5 \% \mathrm{CO}_{2}$ at $37^{\circ} \mathrm{C}$ overnight. Neuro-2a cells were then exposed to $\operatorname{DEHP}(0,1,10$, and $100 \mu \mathrm{M})$ for $24 \mathrm{hr}$ in $1 \mathrm{ml}$ of the experimental medium. Afterwards, the total RNA was extracted and purified using an RNeasy Mini kit (Qiagen, Valencia, CA, USA) and was reverse transcribed into cDNA using the Primescript ${ }^{\mathrm{TM}}$ RT Reagent kit (TaKaRa Bio, Inc., Otsu, Japan). For each sample, the total RNA (500 ng) was reverse transcribed to first-strand cDNA in a final volume of $20 \mu$ l of PrimeScript buffer containing PrimeScript RT Enzyme Mix I ( $1 \mu 1)$, random hexamers (100 pmol), and oligo dT primer (50 pmol) for $15 \mathrm{~min}$ at $37^{\circ} \mathrm{C}$. The reaction was terminated by incubation at $85^{\circ} \mathrm{C}$ for $5 \mathrm{sec}$. The reaction mixtures containing cDNA were stored at $-80^{\circ} \mathrm{C}$ until the real-time PCR analysis.

To determine the efficiency of HO-1 siRNA for knockdown of HO-1 gene, we measured the mRNA level of HO-1 after Neuro-2a cells were transfected with HO-1 siRNA. Neuro-2a cells seeded on $0.25 \%$ polyethyleneimine-coated 12 -well plates $\left(10^{5}\right.$ cells/well $)$ were trans- fected with $10 \mathrm{nM}$ of HO-1 siRNA or control siRNA, and total RNA isolation was performed at 2, 3, 4 days after transfection. Total RNA extraction from the cells and cDNA synthesis were performed using the same procedures described above.

The products of the reverse transcription were used to determine the mRNA levels of HO-1 and GAPDH using Light Cycler 1.5 (Roche Diagnostics). To prepare the standard samples for each gene transcript, an equal amount of each cDNA sample was combined and then serially diluted with EASY Dilution (TaKaRa Bio). The cDNA samples were diluted 10-fold in DNase- and RNase-free water. Two microliters of the standards and diluted unknown samples were amplified in a $20-\mu 1$ reaction mixture containing $200 \mathrm{nM}$ of each primer and $10 \mu 1$ of $2 \times$ SYBR Premix Ex Taq (TaKaRa Bio). The conditions for the real-time PCR were an initial step to activate Taq polymerase for $10 \mathrm{sec}$ at $95^{\circ} \mathrm{C}$, followed by 50 cycles at $95^{\circ} \mathrm{C}$ for $5 \mathrm{sec}$ for denaturation and $60^{\circ} \mathrm{C}$ for $20 \mathrm{sec}$ for annealing and extension, with a temperature transition rate of $20^{\circ} \mathrm{C} / \mathrm{sec}$. After real-time PCR, a melting curve analysis was performed to confirm the specificity of the PCR products. The melting curve for the PCR products targeting each gene transcript showed a single peak (data not shown).

The amount of mRNAs for HO-1 gene in each sample was normalized by dividing each value with the amount of GAPDH mRNA in the same sample. The normalized mRNA levels of HO-1 were then expressed relative to that of the control ( $0 \mu \mathrm{M}$ DEHP) or intact group without transfection of siRNA, which were set at $100 \%$ for each experiment. The percentage was averaged from six or three independent experiments.

\section{Statistical analyses}

A two-way factorial analysis of variance (ANOVA) for repeated measures was used to examine the effects of DEHP exposure on the temporal changes in the ECFP/ Venus emission ratio among the groups. A one-way ANOVA was performed to determine the differences in the mean of the ECFP/Venus emission ratio and mRNA expression level of HO-1 among groups. When significant overall effects were detected using a one-way ANOVA, the Fisher protected least significant difference test was performed as a post-hoc analysis. In the post hoc analysis, a probability value of $p<0.05$ was considered significant. 
DEHP and neurotoxicity

\section{RESULTS}

\section{Effects of DEHP on cell viability of Neuro-2a cells}

Neuro-2a cells were exposed to various concentrations of DEHP $(0,1,10$, or $100 \mu \mathrm{M})$ for $24 \mathrm{hr}$ and cell viability was assessed using a WST-1 assay (Fig. 1). We found that the viability of Neuro-2a cells was significantly decreased by a high-dose DEHP at $100 \mu \mathrm{M}(p<0.01)$, compared with that of control group $(0 \mu \mathrm{M})$. However, the cell viability of DEHP-exposed groups at lower doses (1 and $10 \mu \mathrm{M})$ did not significantly differ from that of the control group.

\section{Effects of DEHP in SCAT3-expressing Neuro-2a cells}

Live imaging analysis resulted in that the morphology and fluorescence intensities of ECFP and Venus of SCAT3-expressing Neuro-2a cells were altered over time by DEHP exposure at a dose of $100 \mu \mathrm{M}$, but not at 0,1 , or $10 \mu \mathrm{M}$ (Fig. 2; Supplementary material: movies 1-4). In Neuro-2a cells exposed to $100 \mu \mathrm{M}$ of DEHP, cells exhibiting a shrunken morphology and apoptotic body forma-

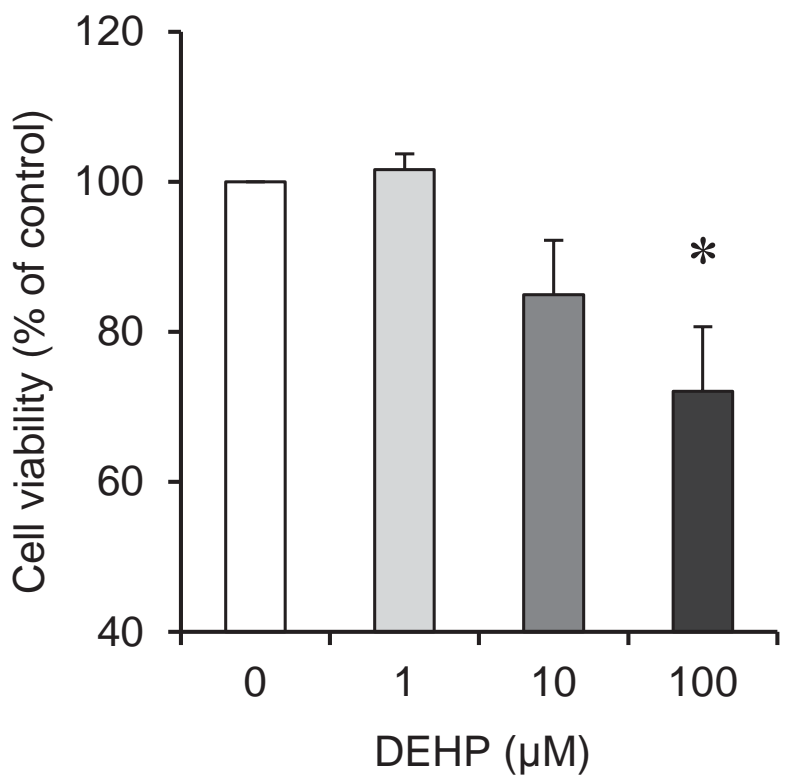

Fig. 1. Effects of DEHP on the viability of Neuro-2a cells. Neuro-2a cells were exposed to the indicated concentration of DEHP for $24 \mathrm{hr}$. The cell viability was expressed relative to the viability of the control group (0 $\mu \mathrm{M}$ DEHP), the value of which was set at $100 \%$. Values are the means \pm S.E.M. of four independent experiments. ${ }^{*} p<0.01$ vs. control group. tion were observed with time after DEHP exposure was started (Fig. 2D). In addition, the fluorescence intensity of ECFP was increased and that of Venus was decreased with time after DEHP exposure, and the signals of both fluorescence proteins were then extremely decreased until the end of live imaging. The temporal pattern of ECFP/Venus emission ratio, which was useful end point to evaluate the effects of chemicals in apoptosis via caspase-3 activation (Koike-Kuroda et al., 2010), in Neuro2a cells exposed to DEHP at $100 \mu \mathrm{M}$ was increased with time compared to Neuro-2a cells of the control group, but not in Neuro-2a cells exposed to lower doses of DEHP (Fig. 3A). A two-way ANOVA for repeated measures indicated that the pattern of the ECFP/Venus emission ratio significantly differed among groups $\left(\mathrm{F}_{3,564}=94.09\right.$, $p<0.001)$ and with time $\left(\mathrm{F}_{46,564}=2.56, p<0.001\right)$. The interactive effect of the main factors, groups and time, was also significant $\left(\mathrm{F}_{138,564}=2.19, p<0.01\right)$.

A one-way ANOVA indicated that there was no significant difference in the mean of ECFP/Venus emission ratio during $1.5-8 \mathrm{hr}$ and $8.5-16 \mathrm{hr}$ after initiation of DEHP exposure (Fig. 3B). However, the mean of the ratio during 16.5-24 hr after DEHP exposure differed significantly among groups $\left(\mathrm{F}_{3,12}=7.01, p<0.05\right)$. A post-hoc analysis showed that the mean of ECFP/Venus emission ratio during 16.5-24 hr after DEHP exposure was significantly increased by high-dose DEHP at $100 \mu \mathrm{M}$, but not by lower doses of DEHP at 1 or $10 \mu \mathrm{M}$, compared to the control group (Fig. 3B).

\section{Effects of DEHP in HO-1 gene expression of Neuro-2a cells}

Live imaging analysis showed that DEHP increased the ECFP/Venus emission of SCAT3-expressing Neuro-2a cells until $24 \mathrm{hr}$ after DEHP exposure was initiated. We therefore measured the mRNA levels of HO-1 in Neuro2a cells $24 \mathrm{hr}$ after DEHP exposure (Fig. 4). A one-way ANOVA indicated that there was significant difference in the mRNA level of HO-1 among groups $\left(\mathrm{F}_{3,20}=11.63\right.$, $p<0.01)$. A post-hoc analysis showed that the mRNA level of HO-1 in Neuro-2a cells exposed to DEHP at $100 \mu \mathrm{M}$ was significantly higher than that of the control group. However, no significant increase of the HO-1 mRNA levels in Neuro-2a cells exposed to DEHP at 1 or $10 \mu \mathrm{M}$ was observed compared to that of the control group.

\section{Effect of HO-1 gene knockdown in DEHP- exposed Neuro-2a cells}

To confirm the efficiency of HO-1 siRNA for gene knockdown, we measured the mRNA level of HO-1 in Neuro-2a cells at 2 to 4 days after cells were transfect- 


\section{(A) Control}

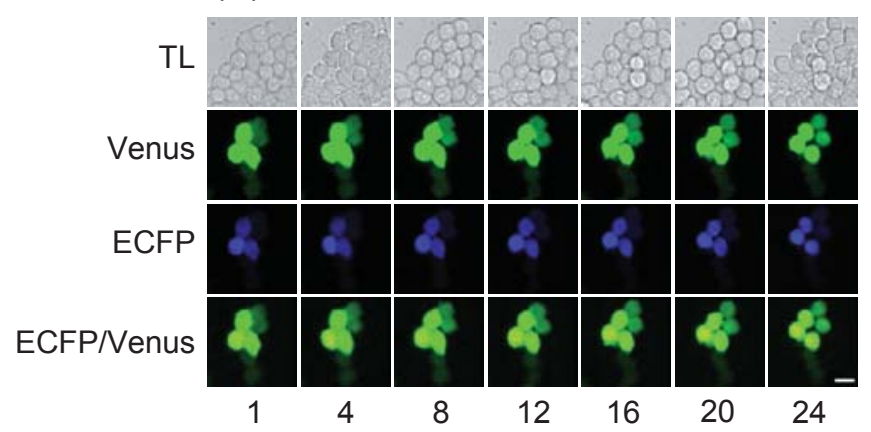

(C) DEHP $10 \mu \mathrm{M}$

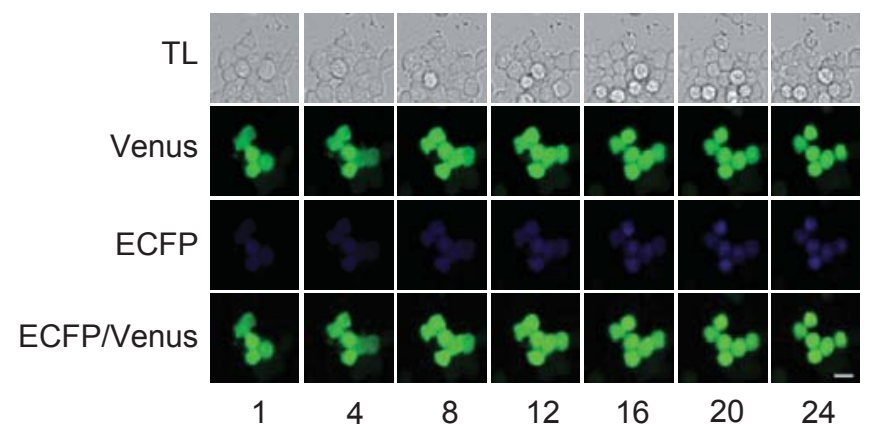

\section{(B) DEHP $1 \mu \mathrm{M}$}

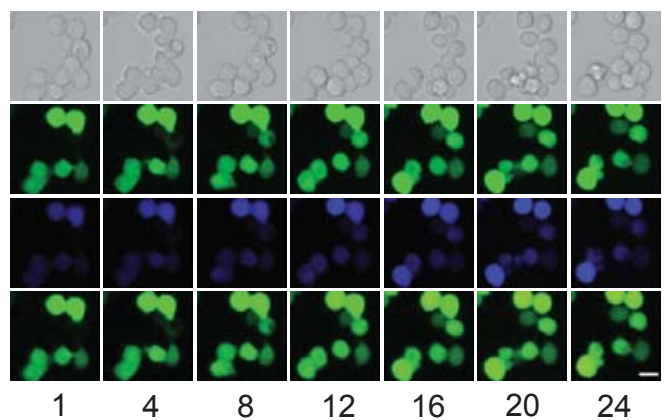

(D) DEHP $100 \mu \mathrm{M}$

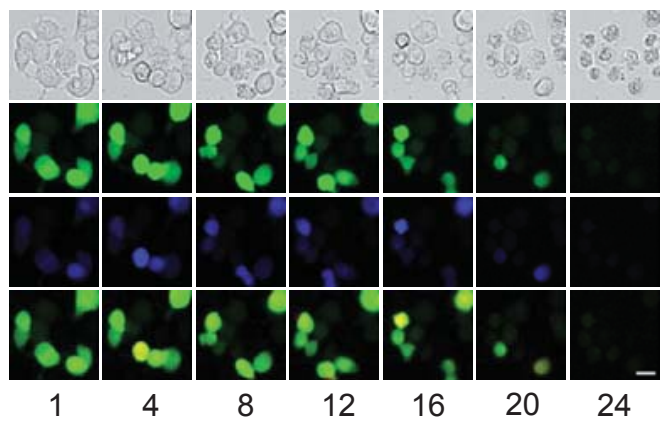

Time after initiation of DEHP exposure (hr)

Fig. 2. Time-lapse images of SCAT3-expressing Neuro-2a cells, which were captured using transmitted light (TL), emissions of Venus and ECFP, and images of Venus and ECFP were merged after the original fluorescent colors were converted into pseudo colors (Venus: yellow-green to green; ECFP: cyan blue to red). Digital images were obtained from SCAT3-expressing Neuro-2a cells exposed to DEHP at concentrations of $0 \mu \mathrm{M}$ (A), $1 \mu \mathrm{M}$ (B), $10 \mu \mathrm{M}$ (C), or $100 \mu \mathrm{M}$ (D) from $1 \mathrm{hr}$ to $24 \mathrm{hr}$ after initiation of DEHP exposure. Scale bars indicate $20 \mu \mathrm{m}$.

ed with HO-1 siRNA. The mRNA level of HO-1 was significantly reduced when Neuro-2a cells were transfected with HO-1 siRNA and cultured for 2, 3, or 4 days after transfection (Fig. 5). On the other hand, there was no significant effect of control siRNA in the HO-1 mRNA level. The HO-1 mRNA level in Neuro-2 cells transfected with HO-1 siRNA was approximately $40 \%$ of Neuro- 2 a cells without siRNA transfection (intact group), and the reduced level was not changed with days after transfection. Accordingly, Neuro-2a cells were kept for 2 or 3 days after transfection with HO-1 siRNA and then used for live imaging analysis to examine the effects of HO-1 gene knockdown in DHEP-induced apoptosis. Live imaging analysis were carried out using $100 \mu \mathrm{M}$ of DEHP, because ECFP/Venus emission in SCAT3-expresing Neuro-2a cells was significantly increased by exposure to DEHP at $100 \mu \mathrm{M}$ but not at 1 or $10 \mu \mathrm{M}$ (Fig. 3).

The morphology and fluorescent intensities of ECFP and Venus were not drastically changed with time in SCAT-3 expressing Neuro-2a cells, which were transfected with control siRNA or HO-1 siRNA and were not exposed to DEHP (Fig. 6A and B; Supplementary material: movies 5 and 6). However, in SCAT3-expressing Neuro-2a cells transfected with control siRNA, the morphology and fluorescence intensities of ECFP and Venus of the cells were altered with time after DEHP exposure (Fig. 6C; Supplementary material: movie 7). The shrinkage of cells and apoptotic body formation were observed in the group of SCAT3-expressing and control siRNAtransfected Neuro-2a cells with time after DEHP exposure. The fluorescence intensity of ECFP was increased and that of Venus was decreased with time after DEHP exposure, and the signals of ECFP and Venus were drastically reduced until the end of live imaging. On the other hand, the morphology and fluorescent signal intensities of ECFP and Venus did not change with DEHP exposure, 
DEHP and neurotoxicity

(A)

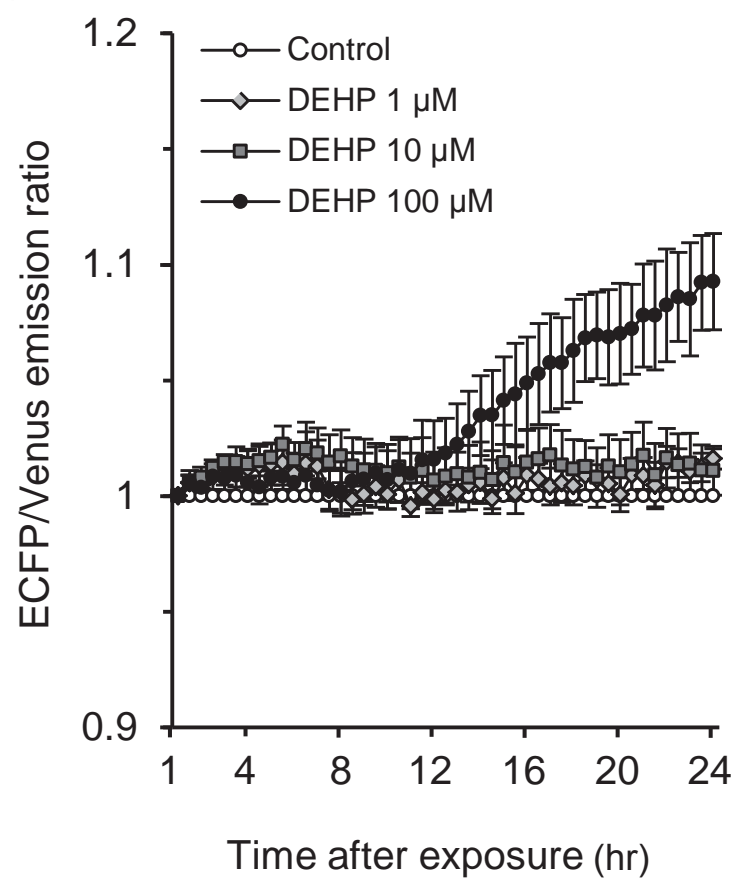

(B)

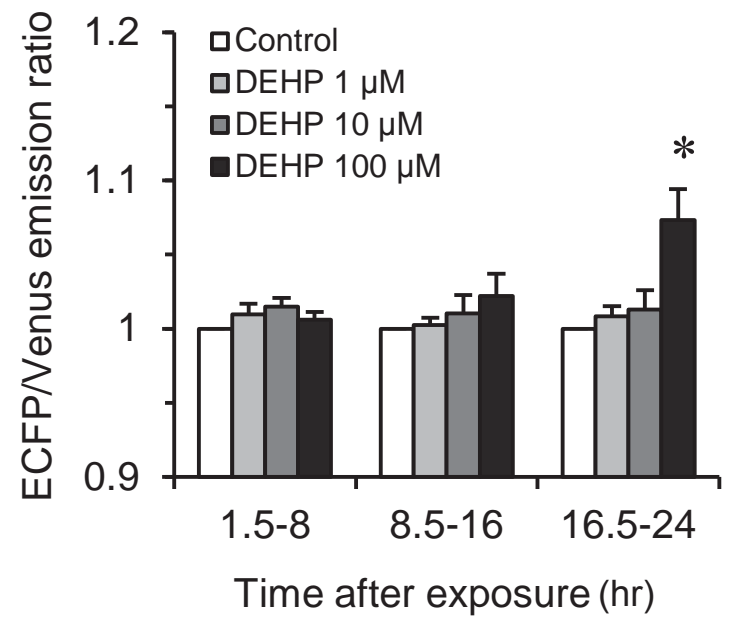

Fig. 3. Effects of DEHP on apoptosis of SCAT3-expressing Neuro-2a cells. (A) Temporal changes in the ECFP/ Venus emission ratio of four different groups of DEHP exposure $(0,1,10$, or $100 \mu \mathrm{M})$. The ratio at each time point was calibrated with the ratio at the same time in the control group, which was set at 1 . (B) The means of the ECFP/Venus emission ratio calculated for 1.5-8 hr, $8.5-16 \mathrm{hr}$, or $16.5-24 \mathrm{hr}$ after initiation of DEHP exposure. The values were the mean \pm S.E.M. of four independent experiments. ${ }^{*} p<0.05$ vs. control group.

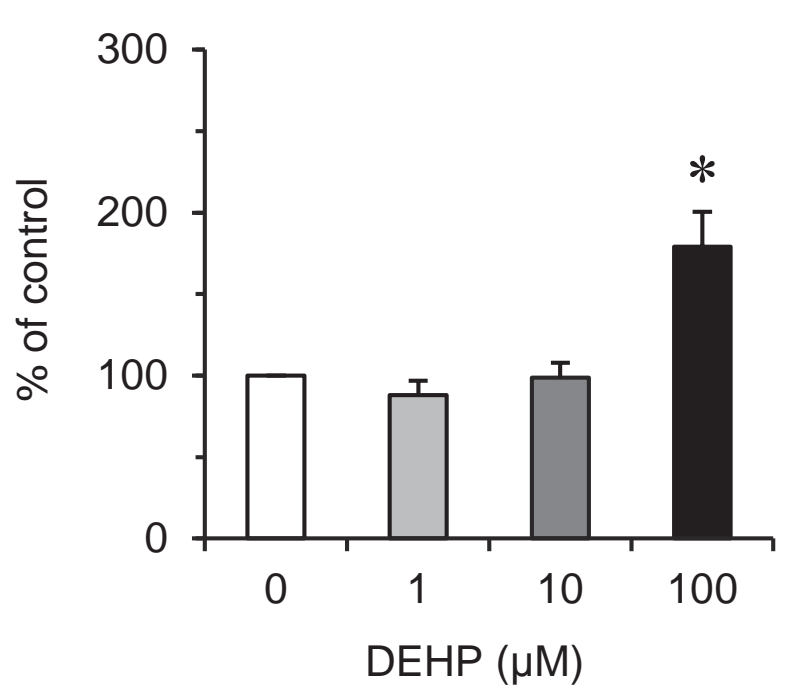

Fig. 4. Effects of DEHP on the mRNA level of hemeoxygenase-1 (HO-1) in Neuro-2a cells. Cells were exposed to DEHP $(0,1,10$, or $100 \mu \mathrm{M})$ for $24 \mathrm{hr}$. The mRNA levels of HO-1 were normalized by dividing them with the mRNA level of GAPDH. The obtained values are presented as the percent of the value in the control. Values are the means \pm S.E.M. of six independent experiments. ${ }^{*} p<0.05$ vs. control group $(0 \mu \mathrm{M}$ DEHP $)$.

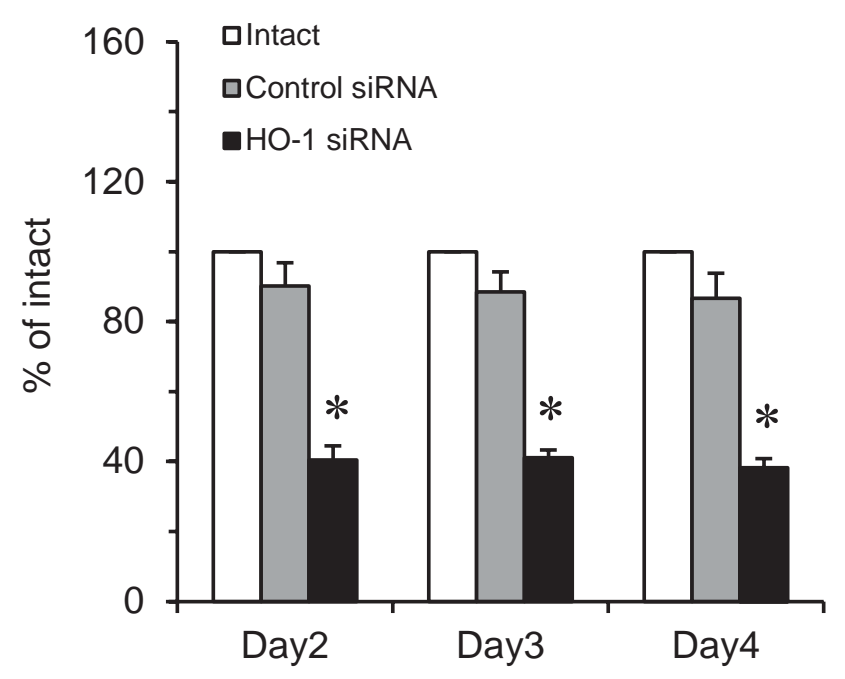

Fig. 5. Effects of HO-1 siRNA on the mRNA level of HO-1 in Neuro-2a cells. Cells were transfected with $10 \mu \mathrm{M}$ of control siRNA or HO-1 siRNA and were cultured for 2, 3, or 4 days. The mRNA level of HO-1 was normalized to the mRNA level of GAPDH and was presented as the percent of the value in the intact group, which was not transfected with any siRNA. Values are the means \pm S.E.M. of three independent experiments. $* p<0.05$ vs. intact group. 


\section{(A) Control siRNA}

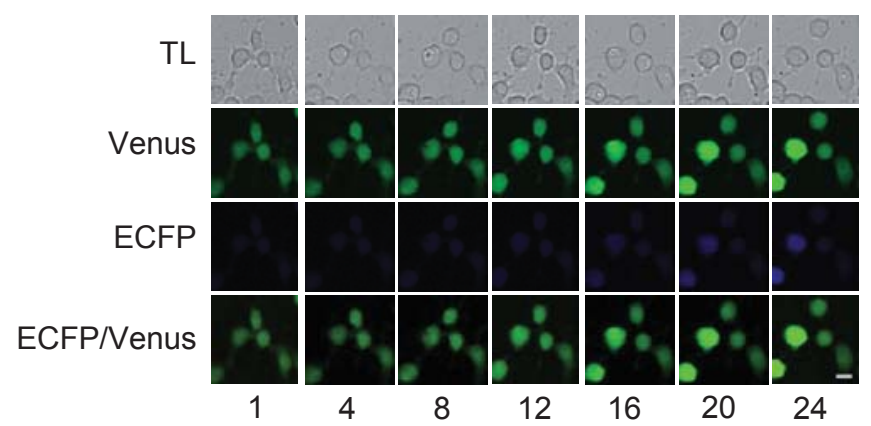

(C) Control siRNA + DEHP

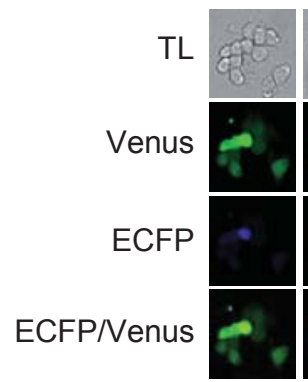

1

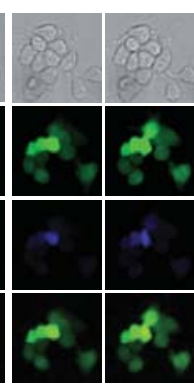

4

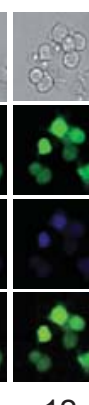

12

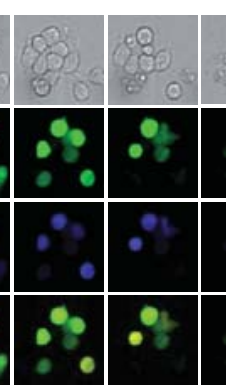

16
(B) HO-1 siRNA

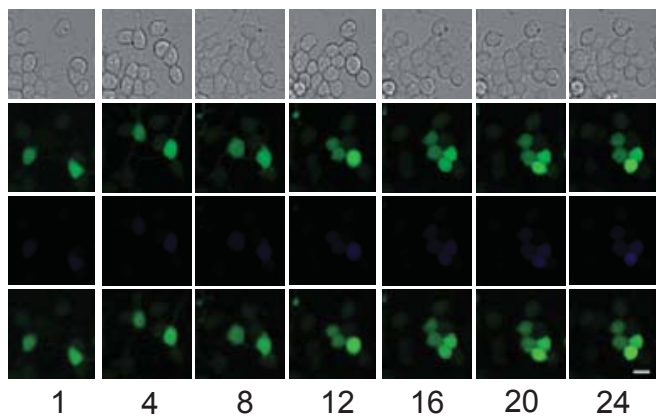

(D) HO-1 siRNA + DEHP

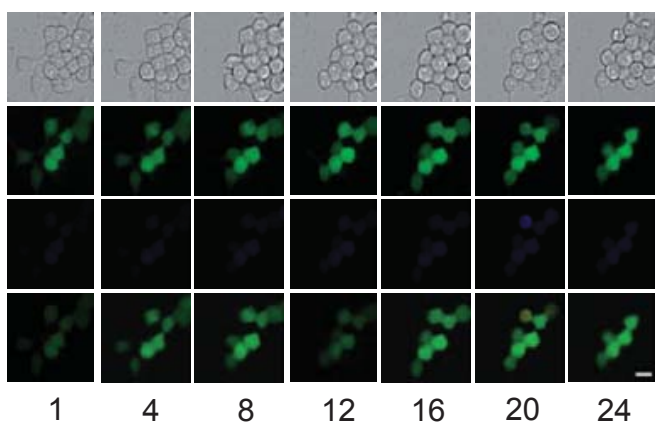

Time after initiation of DEHP exposure (hr)

Fig. 6. Time-lapse images of SCAT3-expressing Neuro-2a cells, which were captured using transmitted light (TL), emissions of Venus and ECFP, and images of Venus and ECFP were merged after the original fluorescent colors were converted into pseudo colors (Venus: yellow-green to green; ECFP: cyan blue to red). Digital images were obtained from SCAT3-expressing Neuro-2a cells pre-treated with control siRNA or HO-1 siRNA, which were exposed to DEHP at a concentration of $0 \mu \mathrm{M}(\mathrm{A}$ and $\mathrm{B})$ or $100 \mu \mathrm{M}(\mathrm{C}$ and $\mathrm{D})$ for $24 \mathrm{hr}$. Scale bars indicate $20 \mu \mathrm{m}$.

when SCAT3-expressing Neuro-2a cells were transfected with HO-1 siRNA (Fig. 6D; Supplementary material: movie 8).

A two-way ANOVA for repeated measures indicated that the temporal pattern of the ECFP/Venus emission ratio of SCAT3-expressing Neuro-2a cell significantly differed among groups with or without DEHP exposure and HO-1 siRNA transfection $\left(\mathrm{F}_{3,564}=138.92, p<0.01\right)$. The pattern of ECFP/Venus emission ratio of SCAT3-expressing Neuro-2a cell transfected with control siRNA was increased with time by DEHP exposure (Fig. 7A). However, the ratio of SCAT3-expressing Neuro-2a cells transfected with HO-1 siRNA was not changed with time by DEHP exposure.

There was no significant difference in the mean of ECFP/Venus emission ratio during 1.5-8 hr after DEHP exposure (Fig. 7B). However, significant differences among experimental groups were found in the mean of
ECFP/Venus emission ratio of SCAT3-expressing Neuro-2a cells during 8.5-16 hr $\left(\mathrm{F}_{3,12}=5.41, p<0.05\right)$ and 16.5-24 hr $\left(\mathrm{F}_{3,12}=5.87, p<0.05\right)$ after DHEP exposure. The mean values of SCAT3-expressing Neuro-2a cells, which were transfected with control siRNA, were significantly increased by DEHP during 8.5-16 hr and 16.5-24 hr after DHEP exposure. However, in SCAT3-expressing and HO-1 siRNA-transfected Neuro-2a cells, DEHP did not increase the mean of ECFP/Venus emission ratio.

\section{DISCUSSION}

It has been demonstrated that DEHP exhibits developmental neurotoxicity (Testa et al., 2012; Cho et al., 2010; Masuo et al., 2004), although the mechanisms, by which DEHP adversely affects the developing brain, are not well understood. DEHP are also detected in the blood of umbilical cord of newborns (Latini et al., 2003), indicat- 
DEHP and neurotoxicity

(A)

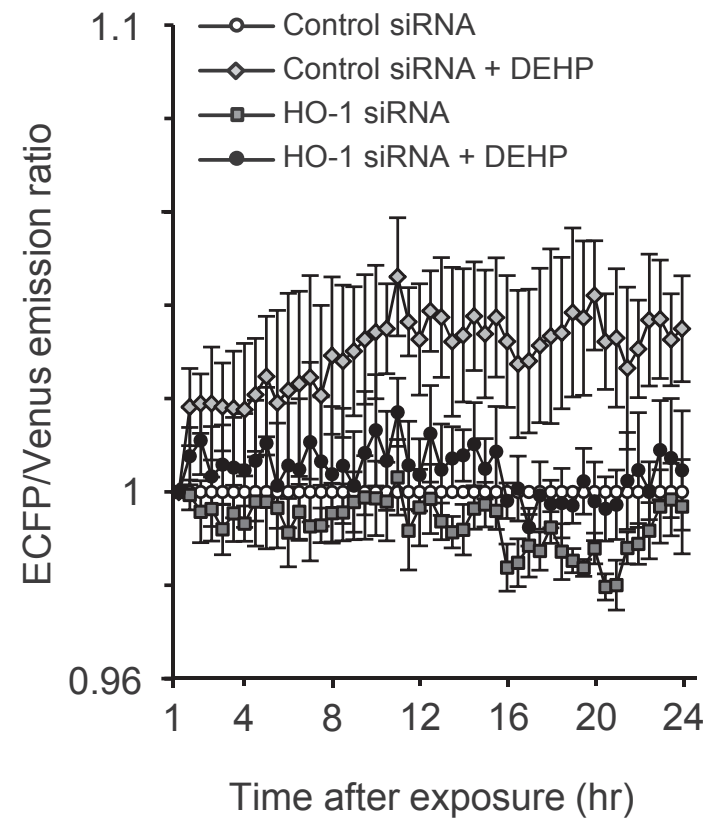

(B)

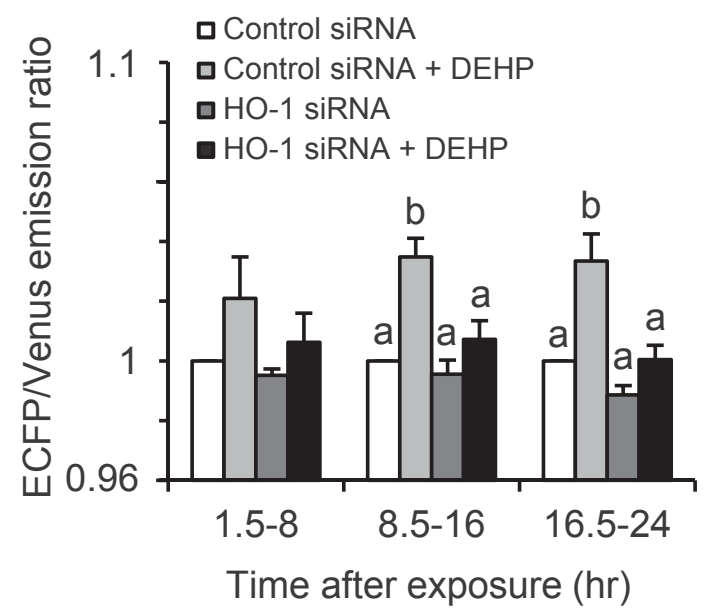

Fig. 7. Effects of HO-1 gene knockdown on DEHP-induced apoptosis in SCAT3-expressing Neuro-2a cells. (A) Temporal changes in the ECFP/Venus emission ratio of SCAT3-expressing Neuro-2a cells. The ratio at each time point was calibrated with the ratio at the same time point in the group treated with control siRNA and $0 \mu \mathrm{M}$ DEHP, which was set at 1 . (B) The mean of ECFP/Venus emission ratio of SCAT3-expressing Neuro-2a cells transfected with control siRNA or HO-1 siRNA for $1.5-8 \mathrm{hr}, 8.5-16 \mathrm{hr}$, or $16.5-24 \mathrm{hr}$ after initiation of DEHP exposure $(0$ or $100 \mu \mathrm{M})$. Values (mean \pm S.E.M. of four independent experiments) with difference characters were significantly $(p<0.05)$ different from each other of the same time. ing that DEHP can pass through the placenta and enter the body of fetuses. Newborns who had contact with plastic medical devices containing DEHP are exposed to the chemical at higher levels, because significantly higher concentrations of DEHP metabolites are detected in the urine samples (Koch et al., 2006). These indicate the risk of DEHP exposure affecting the brain of fetuses and infants. DEHP may directly affect neurons of the developing brain, and then neurons exposed to DEHP may die from apoptosis, because DEHP and its metabolites MEHP activate caspase-3 and induce apoptosis in cultured neuronal cells (Lin et al., 2011; Lim et al., 2009).

Recently, DEHP at a concentration of $40-80 \mu \mathrm{M}$, but not at a concentration of $0.1-10 \mu \mathrm{M}$, was reported to decrease the cell viability and to induce apoptosis in Neuro-2a cells (Lin et al., 2011). In the present study, we showed a decrease in the cell viability of Neuro-2a cells in response to DEHP exposure at a dose of $100 \mu \mathrm{M}$ and confirmed that the viability of Neuro-2a cells was not changed by DEHP exposure at doses less than $10 \mu \mathrm{M}$. We next performed live imaging of SCAT3-expressing Neuro2a cells to evaluate DEHP neurotoxicity and to determine the mode of toxic action of DEHP. SCAT3 is an indicator having caspase-3 cleavage sequence between two fluorescent proteins ECFP and Venus (Takemoto et al., 2003). The usefulness of FRET technology using SCAT3 for monitoring caspase-3 activation in living cells has been well documented (Takemoto et al., 2007; Kuranaga et al., 2006; Kanuka et al., 2005; Takemoto et al., 2003). In addition, we previously showed that live imaging analysis of SCAT3-expressing cultured cells enable screening of the cytotoxicity of chemicals within a short time and with high accuracy compared to other in vitro method for testing cell viability and/or cell death (KoikeKuroda et al., 2010). In the results of our present study, the morphology of SCAT3-expressing Neuro-2a cells and the ECFP/Venus emission ratio indicating the activity of caspase-3 were not changed until $12 \mathrm{hr}$ after exposure to DEHP at any dose was started. However, the temporal pattern of ECFP/Venus emission ratio was started to increase significantly by $100 \mu \mathrm{M}$ DEHP, but not at 1 and $10 \mu \mathrm{M}$, at $15 \mathrm{hr}$ after initiation of DEHP, although the significant increase in the mean of the ECFP/Venus emission ratio was only detected during 16.5 and $24 \mathrm{hr}$ after cells were exposed to $100 \mu \mathrm{M}$ of DEHP. Moreover, apoptotic morphological changes such as shrinkage of the cells and apoptotic body formation were observed with time, especially started from $15 \mathrm{hr}$ after DEHP exposure at $100 \mu \mathrm{M}$. These results suggest that the initiation of apoptosis via caspase-3 activation was started at $15 \mathrm{hr}$ after DEHP exposure. At the end of live imaging, the flu- 
orescent intensities of both ECFP and Venus were drastically decreased in Neuro-2a cells exposed to $100 \mu \mathrm{M}$ of DEHP. We previously reported that the fluorescent intensities of ECFP and Venus were decreased after increasing the ECFP/Venus emission ratio in PC12 cells, when the cell showed abnormal morphology by exposure to sodium arsenite (Koike-Kuroda et al., 2010). It is possible that such changes in the fluorescent signals of SCAT3 are due to degradation of SCAT3 proteins accompanied with apoptotic cell death, although it remains to be elucidated. Taken together, the present study showed that exposure to high-dose DEHP at $100 \mu \mathrm{M}$, but not at lower doses less than $10 \mu \mathrm{M}$, stimulates the activity of caspase- 3 in Neuro-2a cells within $24 \mathrm{hr}$ after exposure, followed by death of cell until $24 \mathrm{hr}$ after exposure. A report has demonstrated that caspase- 3 activity in Neuro-2a cells is stimulated by 48 -hr exposure to DEHP at 40 or $80 \mu \mathrm{M}$ but not at less than $10 \mu \mathrm{M}$ (Lin et al., 2011). The results of our present study support the data of this report and suggest that live imaging of SCAT3-expressing cells can detect the significant effect of DEHP in the activity of casepase-3 with shorter period. Moreover, our study showed that only high-dose DEHP exposure reduced the cell viability and induced apoptosis in Neuro-2a cells, which is consistent and supports our previous in vivo study in which only high level of DEHP induces a neuroinflammatory response in the hypothalamus of mice (Win-Shwe et al., 2013).

HO- 1 is known to be an oxidative stress marker that responds to pathological stimulation, including toxin exposure. For example, the expression of HO-1 in the brain and in vitro cultured neuronal cells is induced by sodium arsenite (Imuta et al., 2007; Kietzmann et al., 2003; Elbirt et al., 1998), cadmium (Alam et al., 2000), and lipopolysacchrides (Hambleton et al., 1996; Han et al., 1994). In addition, we recently reported that DEHP exposure increases the mRNA level of HO- 1 in the hypothalamus of ovalbumin-immunized allergic mice (Win-Shwe et al., 2013). DEHP is known to decrease the cellular levels of antioxidants (Manojkumar et al., 1998) and increases lipid peroxidation (Santhosh et al., 1998). Taken together, it is presumable that DEHP exposure elicits oxidative stress with inducing HO-1 expression. Several lines of evidence indicate that HO-1 has dual effects under oxidative stress (Suttner and Dennery, 1999; Suttner et al., 1999; da Silva et al., 1996). It has been well demonstrated that HO-1 has cytoprotective and antiinflammatory actions in a variety of tissues and cells (Ryter et al., 2007; Grosser et al., 2003; Otterbein et al, 1995). On the other hand, it is also known that HO-1 mediates adverse effects of oxidative stress. Accumu- lation of free iron that is produced by HO- 1 is associated with induction of apoptosis via caspase-3 activation in neurons (Hsieh et al., 2008a, 2008b). In the present study, we found that high-dose DEHP exposure significantly increased the mRNA level of HO-1 in Neuro-2a cells. It has been demonstrated that DEHP activates caspase- 3 and induced caspse-3-dependent apoptosis in Neuro-2 cells (Lin et al., 2011). In this context, it raises the possibility that HO-1, the expression of which is induced by DEHP, is involved in the mechanisms responsible for DEHP-induced apoptosis. To evaluate this possibility, we further performed live imaging analysis of SCAT3-expressing Neuro-2a cells and examined the effects of HO-1 gene knockdown in DEHP-induced apoptosis. As the results of analysis, shrinkage of cells and apoptotic body formation were found and the ECFP/Venus emission ratio, an indicator of enzymatic activity of caspse-3, was increased with time after SCAT3-expressing Neuro-2a cells were exposed to DEHP without HO-1 siRNA treatment. This result again indicates that DEHP activates the enzymatic activity of caspase-3, followed by induction of apoptosis in Neuro-2a cells. On the other hand, when Neuro-2a cells were treated with HO-1 siRNA to reduce the expression level of HO-1 before DEHP exposure was started, such changes in the morphology and indicator of caspase- 3 activity were not observed. These results indicate that HO-1 mediates the toxic actions of DEHP to activate caspase-3 and that knockdown of the HO-1 gene can protect neuronal cells against DEHP-induced apoptosis.

The data in this study from monitoring of apoptotic cell death of SCAT3-expressing Neuro-2a cells showed that the mean ECFP/Venus emission ratio was significantly increased by $100 \mu \mathrm{M}$ DEHP at $16.5-24 \mathrm{hr}$ after DEHP exposure, although the temporal pattern of ECFP/Venus emission ratio in the DEHP $(100 \mu \mathrm{M})$-exposed group was started to at $12 \mathrm{hr}$ after initiation of exposure, However, in the experiments with siRNA treatment, the temporal pattern of ECFP/Venus emission ratio tended to increase early by DEHP exposure, and the significant effect DEHP on the mean ECFP/Venus emission ratio was detected at 8.5-16 hr and 16.5-24 hr after initiation of DEHP exposure. Possible explanation for the observed difference in the results is the different experimental condition. In the experiments of siRNA treatments, we carried out repeated transfection to Neuro-2a cells with SCAT3 and siRNA, while only one transfection procedure for SCAT3 was done in the first experiment. The repeated transfection procedures with different transfection reagents may induce the changes in the cell viability of Neuro-2a cells. Moreover, the cytotoxicity of transfection reagent and siRNA itself should also be considered in this con- 
DEHP and neurotoxicity

text because we maintained Neuro-2a cells in the medium containing the transfection complex for 2 days to achieve the sufficient suppression of the target gene. Studies have shown that siRNA treatment can generate the measurable changes in cellular function and viability in a target-independent manner (Fedorov et al., 2006; Lin et al., 2005). These results indicate that differences in the experimental condition may produce the different sensitivities of cells to toxic chemicals.

It has recently been demonstrated that DEHP increases the production of reactive oxygen species (ROS), a biomarker of oxidative stress, in primary neurons and astrocytes (Wu et al., 2013). This suggests a possibility that oxidative stress-mediated signaling pathways appear to be involved to some extent in the upregulation of HO-1 expression in DEHP-induced neurotoxicity. In fact, the increased ROS production is reportedly known to induce HO-1 expression through activation of nuclear-factor-E2related factor-2 (Nrf-2), a transcriptional factor, in culture retinal neurons (Koriyama et al., 2013). Moreover, activation of Nrf-2 in combination with activating Peroxisome proliferator-activated receptor- $\gamma$ (PPAR $\gamma)$, a ligandactivated transcription factor, is known to contribute the induction of HO-1 expression under oxidative stress condition (Kang et al., 2005). Several studies revealed that PPAR $\gamma$ increases the expression of HO-1 in human hepatocytes (Wang et al., 2011), human vascular endothelial and smooth muscle cells (Krönke et al., 2007), and rat motor neurons (Park et al., 2007). Analysis of the human HO-1 promoter indicates that HO-1 is a direct PPAR-target gene, the transcription of which is regulated by PPAR $\alpha$ and PPAR $\gamma$ via PPAR-responsive elements (Krönke et al., 2007). These studies indicate that HO-1 is transcriptionally regulated by PPARs especially by PPAR $\gamma$. Interestingly, DEHP exposure stimulates the induction of PPAR $\gamma$ expression in the testes of rats (Ryu et al., 2007) and Neuro-2a cells (Lin et al., 2011). Furthermore, cleavage of pro-caspase- 3 for activating caspase- 3 are caused by DEHP exposure in Neuor-2a cells, and this cleavage occurring with DEHP exposure is suppressed by a PPAR $\gamma$ antagonist and enhanced by a PPAR $\gamma$ agonist (Lin et al., 2011), suggesting that PPAR $\gamma$ mediates the pro-apoptotic actions of DEHP. Taking this evidence together, the mechanisms of DEHP-induced apoptosis in Neuro-2a cells can be speculated that DEHP firstly activates transcriptional factors such as Nrf-2 and PPAR $\gamma$, followed by induction of the transcription of target gene including HO-1, and HO- 1 then activates caspase- 3 and induces apoptosis in Neuro-2a cells. Thus, HO- 1 could be a downstream molecule of transcription factors Nrf-2 and PPAR $\gamma$ in the signal cascade of DEHP-induced apoptosis. HO-1 cata- lyzes the degradation of heme to biliverdin, iron, and $\mathrm{CO}$. Accumulation of free iron and $\mathrm{CO}$ produced by $\mathrm{HO}-1$ in astrocytes and neurons causes intracellular oxidative stress and mitochondrial damage, which appears to contribute the pathogenesis of neurodegenerative diseases (Justicia et al., 2008; Schipper et al., 2000, 1998). One report showed that increased formation of iron by HO-1 is followed by activation of caspase- 3 and induction of apoptosis (Hsieh et al., 2008a). Hence, intercellular free iron that is excessively produced by HO-1 may be partially involved in the downstream cascade of DEHP-induced apoptosis, although further studies are needed to clarify it.

In conclusion, we investigated the effects of DEHP exposure on apoptosis and clarified the role of HO-1 in DEHP-induced apoptosis of Neuro-2a cells. The present study demonstrated that high-dose of DEHP exposure induced the neurotoxic effects in neurons. We found that the expression of HO-1 in Neuro-2a cells was significantly induced by high-dose DEHP. Live imaging analysis of Neuro-2a cells confirmed that high-dose DEHP significantly activates the enzymatic activity of caspase-3, followed by induction of apoptotic cell death in Neuro$2 \mathrm{a}$ cells. These results suggest raising the awareness of DEHP usage such as neonatal exchange transfusion with PVC-made catheters that can cause high-level exposure to DEHP. In this study, we also found that the toxic effect of DEHP on apoptosis of Neuro-2a cells was nullified by knockdown of the HO-1 gene. This indicates that HO-1 is involved in DEHP-induced apoptosis through activation of caspase-3 in Neuro-2a cells. This is the first study showing the involvement of HO-1 in the mechanism of DEHP-induced neurotoxicity and apoptosis. Therefore, HO- 1 could be a potential therapeutic target for treatment of DEHP-induced neurotoxicity and its related neurological disorders.

\section{ACKNOWLEDGEMENTS}

This study was supported in part by a grant from the Japan Chemical Industry Association (JCIA) Long-range Research Initiative (LRI) to S.T., by a Grant-in-Aid for Scientific Research from the Ministry of Education, Science, Culture and Sports of Japan (23310043 to S.T. and 25340066 to T.T.W.S.) and by a grant from the National Institute for Environmental Studies, Japan (1115AA082 and 1013BD001) to T.T.W.S. We thank Dr. M. Miura (University of Tokyo, Tokyo, Japan) for providing the pcDNA-SCAT3 vector. 


\section{REFERENCES}

Alam, J., Wicks, C., Stewart, D., Gong, P., Touchard, C., Otterbein, S., Choi, A.M., Burow, M.E. and Tou, J. (2000): Mechanism of heme oxygenase-1 gene activation by cadmium in MCF-7 mammary epithelial cells. Role of p38 kinase and Nrf2 transcription factor. J. Biol. Chem., 275, 27694-27702.

Arcadi, F.A., Costa, C., Imperatore, C., Marchese, A., Rapisarda, A., Salemi, M., Trimarchi, G.R. and Costa, G. (1998): Oral toxicity of bis(2-ethylhexyl) phthalate during pregnancy and suckling in the Long-Evans rat. Food Chem. Toxicol., 36, 963-970.

Aung, K.H., Kurihara, R., Nakashima, S., Maekawa, F., Nohara, K., Kobayashi, T. and Tsukahara, S. (2013): Inhibition of neurite outgrowth and alteration of cytoskeletal gene expression by sodium arsenite. Neurotoxicology, 34, 226-235.

Cho, S.C., Bhang, S.Y., Hong, Y.C., Shin, M.S., Kim, B.N., Kim, J.W., Yoo, H.J., Cho, I.H. and Kim, H.W. (2010): Relationship between environmental phthalate exposure and the intelligence of school-age children. Environ. Health Perspect., 118, 10271032.

da Silva, J.L., Morishita, T., Escalante, B., Staudinger, R., Drummond, G., Goligorsky, M.S., Lutton, J.D. and Abraham, N.G. (1996): Dual role of heme oxygenase in epithelial cell injury: contrasting effects of short-term and long-term exposure to oxidant stress. J. Lab. Clin. Med., 128, 290-296.

Elbirt, K.K., Whitmarsh, A.J., Davis, R.J. and Bonkovsky, H.L. (1998): Mechanism of sodium arsenite-mediated induction of heme oxygenase-1 in hepatoma cells. Role of mitogen-activated protein kinases. J. Biol. Chem., 273, 8922-8931.

Fedorov, Y., Anderson, E.M., Birmingham, A., Reynolds, A., Karpilow, J., Robinson, K., Leake, D., Marshall, W.S. and Khvorova, A. (2006): Off-target effects by siRNA can induce toxic phenotype. RNA, 12, 1188-1196.

Grosser, N., Abate, A., Oberle, S., Vreman, H.J., Dennery, P.A., Becker, J.C., Pohle, T., Seidman, D.S. and Schröder, H. (2003): Heme oxygenase-1 induction may explain the antioxidant profile of aspirin. Biochem. Biophys. Res. Commun., 308, 956-960.

Hambleton, J., Weinstein, S.L., Lem, L. and DeFranco, A.L. (1996): Activation of c-Jun N-terminal kinase in bacterial lipopolysaccharide-stimulated macrophages. Proc. Natl. Acad. Sci. USA, 93, 2774-2778

Han, J., Lee, J.D., Bibbs, L. and Ulevitch, R.J. (1994): A MAP kinase targeted by endotoxin and hyperosmolarity in mammalian cells. Science, 265, 808-811.

Hsieh, C.H., Jeng, S.F., Hsieh, M.W., Chen, Y.C., Rau, C.S., Lu, T.H. and Chen, S.S. (2008a): Statin-induced heme oxygenase-1 increases NF-kappaB activation and oxygen radical production in cultured neuronal cells exposed to lipopolysaccharide. Toxicol. Sci., 102, 150-159.

Hsieh, C.H., Rau, C.S., Hsieh, M.W., Chen, Y.C., Jeng, S.F., Lu, T.H. and Chen, S.S. (2008b): Simvastatin-induced heme oxygenase-1 increases apoptosis of Neuro 2A cells in response to glucose deprivation. Toxicol. Sci., 101, 112-121.

Imuta, N., Hori, O., Kitao, Y., Tabata, Y., Yoshimoto, T., Matsuyama, T. and Ogawa, S. (2007): Hypoxia-mediated induction of heme oxygenase type I and carbon monoxide release from astrocytes protects nearby cerebral neurons from hypoxiamediated apoptosis. Antioxid. Redox. Signal, 9, 543-552.

Justicia, C., Ramos-Cabrer, P. and Hoehn, M. (2008): MRI detection of secondary damage after stroke: chronic iron accumulation in the thalamus of the rat brain. Stroke, 39, 1541-1547.
Kang, K.W., Lee, S.J. and Kim, S.G. (2005): Molecular mechanism of nrf2 activation by oxidative stress. Antioxid. Redox Signal, 7, 1664-1673.

Kanuka, H., Kuranaga, E., Takemoto, K., Hiratou, T., Okano, H. and Miura, M. (2005): Drosophila caspase transduces Shaggy/GSK3 beta kinase activity in neural precursor development. EMBO J., 24, 3793-3806.

Kavlock, R., Boekelheide, K., Chapin, R., Cunningham, M., Faustman, E., Foster, P., Golub, M., Henderson, R., Hinberg, I., Little, R., Seed, J., Shea, K., Tabacova, S., Tyl, R., Williams, P. and Zacharewski, T. (2002): NTP Center for the Evaluation of Risks to Human Reproduction: phthalates expert panel report on the reproductive and developmental toxicity of di(2-ethylhexyl) phthalate. Reprod. Toxicol., 16, 529-653.

Kietzmann, T., Samoylenko, A. and Immenschuh, S. (2003): Transcriptional regulation of heme oxygenase-1 gene expression by MAP kinases of the JNK and p38 pathways in primary cultures of rat hepatocytes. J. Biol. Chem., 278, 17927-17936.

Klinefelter, G.R., Laskey, J.W., Winnik, W.M., Suarez, J.D., Roberts, N.L., Strader, L.F., Riffle, B.W. and Veeramachaneni, D.N. (2012): Novel molecular targets associated with testicular dysgenesis induced by gestational exposure to diethylhexyl phthalate in the rat: a role for estradiol. Reproduction, 144, 747761.

Koch, H.M., Preuss, R. and Angerer, J. (2006): Di(2-ethylhexyl) phthalate (DEHP): human metabolism and internal exposure-an update and latest results. Int. J. Androl., 29, 155-165; discussion 181-155.

Koike-Kuroda, Y., Kakeyama, M., Fujimaki, H. and Tsukahara, S. (2010): Use of live imaging analysis for evaluation of cytotoxic chemicals that induce apoptotic cell death. Toxicol. In Vitro, 24, 2012-2020.

Koriyama, Y., Nakayama, Y., Matsugo, S. and Kato, S. (2013): Protective effect of lipoic acid against oxidative stress is mediated by Keap1/Nrf2-dependent heme oxygenase-1 induction in the RGC-5 cellline. Brain Res., 1499, 145-157.

Krönke, G., Kadl, A., Ikonomu, E., Blüml, S., Fürnkranz, A., Sarembock, I.J., Bochkov, V.N., Exner, M., Binder, B.R. and Leitinger, N. (2007): Expression of heme oxygenase-1 in human vascular cells is regulated by peroxisome proliferator-activated receptors. Arterioscler. Thromb. Vasc. Biol., 27, 1276-1282.

Kuida, K., Zheng, T.S., Na, S., Kuan, C., Yang, D., Karasuyama, H., Rakic, P. and Flavell, R.A. (1996): Decreased apoptosis in the brain and premature lethality in CPP32-deficient mice. Nature, 384, 368-372.

Kuranaga, E., Kanuka, H., Tonoki, A., Takemoto, K., Tomioka, T., Kobayashi, M., Hayashi, S. and Miura, M. (2006): Drosophila IKK-related kinase regulates nonapoptotic function of caspases via degradation of IAPs. Cell, 126, 583-596.

Latini, G., De Felice, C., Presta, G., Del Vecchio, A., Paris, I., Ruggieri, F. and Mazzeo, P. (2003): In utero exposure to di-(2ethylhexyl)phthalate and duration of human pregnancy. Environ. Health Perspect., 111, 1783-1785.

Lim, C.K., Kim, S.K., Ko, D.S., Cho, J.W., Jun, J.H., An, S.Y., Han, J.H., Kim, J.H. and Yoon, Y.D. (2009): Differential cytotoxic effects of mono-(2-ethylhexyl) phthalate on blastomere-derived embryonic stem cells and differentiating neurons. Toxicology, 264, 145-154.

Lin, C.H., Chen, T.J., Chen, S.S., Hsiao, P.C. and Yang, R.C. (2011): Activation of Trim 17 by PPAR $\gamma$ is involved in di(2-ethylhexyl) phthalate (DEHP)-induced apoptosis on Neuro-2a cells. Toxicol. Lett., 206, 245-251. 
DEHP and neurotoxicity

Lin, X., Ruan, X., Anderson, M.G., McDowell, J.A., Kroeger, P.E., Fesik, S.W. and Shen, Y. (2005): siRNA-mediated off-target gene silencing triggered by a $7 \mathrm{nt}$ complementation. Nucleic Acids Res., 33, 4527-4535.

Manojkumar, V., Padmakumaran Nair, K.G., Santhosh, A., Deepadevi, K.V., Arun, P., Lakshmi, L.R. and Kurup, P.A. (1998): Decrease in the concentration of vitamin E in blood and tissues caused by di(2-ethylhexyl) phthalate, a commonly used plasticizer in blood storage bags and medical tubing. Vox. Sang., 75, 139-144.

Masuo, Y., Morita, M., Oka, S. and Ishido, M. (2004): Motor hyperactivity caused by a deficit in dopaminergic neurons and the effects of endocrine disruptors: a study inspired by the physiological roles of PACAP in the brain. Regul. Pept., 123, 225-234.

Mitani, K., Fujita, H., Fukuda, Y., Kappas, A. and Sassa, S. (1993): The role of inorganic metals and metalloporphyrins in the induction of haem oxygenase and heat-shock protein 70 in human hepatoma cells. Biochem. J., 290, 819-825.

Otterbein, L., Sylvester, S.L. and Choi, A.M. (1995): Hemoglobin provides protection against lethal endotoxemia in rats: the role of heme oxygenase-1. Am. J. Respir. Cell Mol. Biol., 13, 595601.

Park, S.W., Yi, J.H., Miranpuri, G., Satriotomo, I., Bowen, K., Resnick, D.K. and Vemuganti, R. (2007): Thiazolidinedione class of peroxisome proliferator-activated receptor gamma agonists prevents neuronal damage, motor dysfunction, myelin loss, neuropathic pain, and inflammation after spinal cord injury in adult rats. J. Pharmacol. Exp. Ther., 320, 1002-1012.

Pettmann, B. and Henderson, C.E. (1998): Neuronal cell death. Neuron, 20, 633-647.

Ryter, S.W., Morse, D. and Choi, A.M. (2007): Carbon monoxide and bilirubin: potential therapies for pulmonary/vascular injury and disease. Am. J. Respir. Cell Mol. Biol., 36, 175-182.

Ryu, J.Y., Whang, J., Park, H., Im, J.Y., Kim, J., Ahn, M.Y., Lee, J., Kim, H.S., Lee, B.M., Yoo, S.D., Kwack, S.J., Oh, J.H., Park, K.L., Han, S.Y. and Kim, S.H. (2007): Di(2-ethylhexyl) phthalate induces apoptosis through peroxisome proliferators-activated receptor-gamma and ERK 1/2 activation in testis of SpragueDawley rats. J. Toxicol. Environ. Health, 70, 1296-1303.

Santhosh, A., Nair, K.G., Arun, P., Deepadevi, K.V., Manojkumar, V., Lakshmi, L.R. and Kurup, P.A. (1998): Effect of DEHP [di(2-ethyl hexyl) phthalate] on lipid peroxidation in liver in rats and in primary cultures of rat hepatocytes. Indian J. Med. Res., 108, 17-23.

Schipper, H.M. (2000): Heme oxygenase-1: role in brain aging and neurodegeneration. Exp. Gerontol., 35, 821-830.

Schipper, H.M., Liberman, A. and Stopa, E.G. (1998): Neural heme oxygenase-1 expression in idiopathic Parkinson's disease. Exp. Neurol., 150, 60-68.

Suttner, D.M. and Dennery, P.A. (1999): Reversal of HO-1 related cytoprotection with increased expression is due to reactive iron. FASEB J., 13, 1800-1809.

Suttner, D.M., Sridhar, K., Lee, C.S., Tomura, T., Hansen, T.N. and Dennery, P.A. (1999): Protective effects of transient HO-1 overexpression on susceptibility to oxygen toxicity in lung cells. Am. J. Physiol., 276, L443-451.

Takemoto, K., Kuranaga, E., Tonoki, A., Nagai, T., Miyawaki, A. and Miura, M. (2007): Local initiation of caspase activation in Drosophila salivary gland programmed cell death in vivo. Proc. Natl. Acad. Sci. USA, 104, 13367-13372.

Takemoto, K., Nagai, T., Miyawaki, A. and Miura, M. (2003): Spatio-temporal activation of caspase revealed by indicator that is insensitive to environmental effects. J. Cell Biol., 160, 235243.

Tanida, T., Warita, K., Ishihara, K., Fukui, S., Mitsuhashi, T., Sugawara, T., Tabuchi, Y., Nanmori, T., Qi, W.M., Inamoto, T., Yokoyama, T., Kitagawa, H. and Hoshi, N. (2009): Fetal and neonatal exposure to three typical environmental chemicals with different mechanisms of action: mixed exposure to phenol, phthalate, and dioxin cancels the effects of sole exposure on mouse midbrain dopaminergic nuclei. Toxicol. Lett., 189, 40-47.

Testa, C., Nuti, F., Hayek, J., De Felice, C., Chelli, M., Rovero, P., Latini, G. and Papini, A.M. (2012): Di-(2-ethylhexyl) phthalate and autism spectrum disorders. ASN Neuro., 4, 223-229.

Wang, X., Wang, Z., Liu, J.Z., Hu, J.X., Chen, H.L., Li, W.L. and Hai, C.X. (2011): Double antioxidant activities of rosiglitazone against high glucose-induced oxidative stress in hepatocyte. Toxicol. In Vitro, 25, 839-847.

Win-Shwe, T.T., Yanagisawa, R., Koike, E., Nitta, H. and Takano, H. (2013): Expression levels of neuroimmune biomarkers in hypothalamus of allergic mice after phthalate exposure. J. Appl. Toxicol., 33, 1070-1078.

Xi, W., Wan, H.T., Zhao, Y.G., Wong, M.H., Giesy, J.P. and Wong, C.K. (2011): Effects of perinatal exposure to bisphenol A and di(2-ethylhexyl)-phthalate on gonadal development of male mice. Environ. Sci. Pollut. Res. Int., 19, 2515-2527.

Wu, Y., Li Ke, Zuo, H., Yuan, Y., Sun, Y. and Yang X. (2013): Primary neuronal-astrocytic co-culture platform for neurotoxicity assessment of di-(2-ethylhexyl) phthalate. J. Environ. Sci., DOI: 10.1016/S1001-0742(13)60504-5.

Yan, Y., Black, C.P. and Cowan, K.H. (2007): Irradiation-induced $\mathrm{G} 2 / \mathrm{M}$ checkpoint response requires ERK1/2 activation. Oncogene, 26, 4689-4698. 